\title{
Evaluation of Matrix-Assisted Laser Desorption Ionization Mass Spectrometry for Polymer Characterization
}

\author{
A. M. Belu, J. M. DeSimone, and R. W. Linton \\ Department of Chemistry, University of North Carolina, Chapel Hill, North Carolina, USA \\ G. W. Lange and R. M. Friedman \\ Monsanto Corporation, St. Louis, Missouri, USA
}

A protocol for the preparation of polymeric samples for time-of-flight matrix-assisted laser desorption ionization mass spectrometry (TOF-MALDI-MS) analysis was developed. Dithranol was identified as a good matrix for polystyrene (PS), and the addition of silver for cationization of molecules was determined to be necessary. Based on this preparative method, low molecular weight samples of other polymers [polyisoprene, polybutadiene, poly(ethylene oxide), poly(methyl methacrylate), and polydimethylsiloxane] were analyzed with molecular weights up to $49 \mathrm{ku}$. The effects of laser intensity were determined to influence the molecular weight distribution of intact oligomers, most significantly for low molecular weight polymers. Linear and reflectron modes of analysis were evaluated; better signal intensity and resolution were obtained in the reflectron mode. The TOF-MALDI-MS measurements are compared with time-of-flight secondary ion mass spectrometry (TOF-SIMS) and gel permeation chromatography (GPC) for the same polymers. The $M_{n}$ values calculated by TOF-MALDI-MS consistently are higher than values calculated by TOF-SIMS for all classes of polymers with molecular weights up to $8 \mathrm{ku}$. The molecular weights of the PS calculated from TOF-MALDI-MS are in good agreement with GPC $( \pm 10 \%)$. The composition of the terminal group on a polymer chain may affect the ion yields. The ion yields of intact oligomers were evaluated as a function of end group composition for both TOF-MALDI-MS and TOF-SIMS. The slight disparity of results between TOF-SIMS and TOF-MALDI-MS for the perfluoroalkyl-terminated PS suggests that the oligomers are desorbed preferentially from the surface in the TOF-SIMS analysis, rather than having an increased ionization probability. (J Am Soc Mass Spectrom 1996, 7, 11-24)

$\Lambda$ $s$ a powerful new method for the analysis of high molecular weight materials, matrix-assisted laser desorption ionization (MALDI) is pertinent to the characterization of polymers. To date, the development of the technique has been directed mainly toward biomolecules; few studies have focused on synthetic polymers. The first examples of polymer characterization by MALDI were presented by Tanaka et al. [1], who introduced the technique in 1988. Distributions of sodium-cationized molecular ions were shown for 4-ku poly(propylene glycol) (PPG) and 20-ku poly(ethylene glycol) (PEG) by using the "ultra fine metal plus liquid matrix method," a matrix that consists of cobalt powder in glycerol. By 1992, a standard preparation of samples for MALDI analysis of biomolecules had been developed by using sinapinic acid as a solid matrix with crystalline regions. Danis et

Address reprint requests to Dr. Richard $W$. Linton, Department of Chemistry, CB\# 3290, University of North Carolina, Chapel Hill, NC 27599-3290. al. [2] were the first to apply this preparation to synthetic polymers. They analyzed poly(acrylic acid) (3 $\mathrm{ku})$ and poly(styrene sulfonic acid) $(200 \mathrm{ku})$, which are polar, water-soluble molecules that have good miscibility with sinapinic acid. The Hillenkamp group [3] provided the first comprehensive study of synthetic polymers by MALDI. Molecular ions were observed for poly(methyl methacrylate) (PMMA) as well as PPG and PEG by using a solid matrix of 2,5-dihydroxybenzoic acid (DHB). Sometimes alkali-metal salt solutions $(\mathrm{NaCl}, \mathrm{LiCl}, \mathrm{KCl})$ were added to increase the yield of cationized species. The capability of this technique to determine accurate molecular weight values was demonstrated for these polymers with molecular weights up to $40 \mathrm{ku}$. Also, the first MALDI analysis of a hydrophobic polymer was shown for polystyrene (PS) with molecular weights up to $80 \mathrm{ku}$ using a liquid matrix of 2-nitrophenyl octyl ether (NPOE) and silver trifluoroacetate (AgTFA) for cationization. Danis and Karr [4] further developed the approach to sample preparation for organic-soluble polymers. The use of 
matrices soluble in organic solvents allowed direct mixing with the organic-soluble polymer and promoted polymer-matrix interaction. Polymers previously had been prone to precipitate out of solution (partially water-based) before the matrix crystallized. Via the organic solvent approach, molecular weight distributions were obtained for samples of PMMA with molecular weights up to $260 \mathrm{ku}$, prepared in a matrix of DHB or in t-3-indoleacrylic acid (IAA) with acetone. This approach also was used to obtain PS (molecular weights of 11.6 and $120 \mathrm{ku}$ ) spectra with the silver metal salt of IAA as a matrix [5]. The sample preparation method of Danis and Karr [4] is indicated to be applicable to a range of polymers, which include polycarbonate, poly(vinyl acetate), and poly(vinyl chloride), but a more detailed review of procedures and results is needed.

As can be seen from the developmental emphasis of matrix-assisted laser desorption ionization mass spectrometry (MALDI-MS) for polymer characterization thus far, the preparation of analyte in a proper matrix plays a crucial role in the viability of the technique. However, each report details a unique sample preparation procedure. No standard protocol has been developed yet because of the diversity of polymeric materials available, the varying instrumental conditions under which the measurements are made, and the ambiguous role the matrix plays in desorption and ionization of the analyte. The analysis of PEG in a matrix of DHB or sinapinic acid is probably the most reproduced polymer sample preparation. This sample preparation is excellent for biomolecules because they are similarly polar and water-soluble. Because most polymers are organic-soluble, the Danis and Karr approach to sample preparation is becoming popular due to its indicated applicability to a range of polymers and its use of a single organic solvent.

Few MALDI-MS results have been shown for hydrocarbon polymers. To find an appropriate matrix and preparation method for these materials is especially difficult because of their nonaqueous and nonpolar nature. Wilkins suggested that hydrocarbon polymers lack an effective site for ionization, and laser desorption thereby yields neutral gas-phase molecules [6]. PS may be the easiest hydrocarbon to ionize because its phenyl functionality allows for a higher ionization probability, especially compared to polyisoprene (PI) and polybutadiene (PBD) (branched polymers with vinylic functionality) and polyethylene (PE) (a completely aliphatic polymer). Ion yields may be increased by the presence of alkali metals, particularly for polar polymers where the heteroatoms $(O, N)$ are the sites of alkali metal attachment. With nonaromatic hydrocarbon polymers, however, difficulties in their ionization remains due to their lack of energetically favorable sites for alkali metal attachment [6].

The goal of this study is to determine optimal MALDI-MS conditions for a broad range of synthetic organic polymers. The development of a highly repro- ducible sample preparation procedure was investigated. At the same time the role of instrumental conditions such as laser power, extraction energy, and analyzer mode (linear, reflectron) were explored. The search for an ideal sample preparation method first focused on hydrocarbon polymers-specifically PS. Hydrocarbons represent a large class of polymeric materials to which MALDI-MS has not been applied yet. The concepts learned by developing a sample preparation procedure for PS were applied toward the development of MALDI-MS for other polymer classes as well. Furthermore, many of the polymers studied have been characterized by using time-of-flight secondary ion mass spectrometry (TOF-SIMS) and gel permeation chromatography (GPC). The results from these techniques allow further assessment of the information generated by MALDI-MS.

\section{Experimental}

Materials. The polymers polystyrene (PS), polyisoprene (PI), polybutadiene (PBD), polyethylene (PE), and polydimethylsiloxane (PDMS) were synthesized by living anionic polymerization at the University of North Carolina at Chapel Hill [7]. Polymethylmethacrylate (PMMA) was obtained from American Polymer Standards Corp. (Mentor, $\mathrm{OH}$ ) and poly(ethylene oxide) (PEO) was obtained from Polymer Labs (Church Stretton, UK). The matrices 2-nitrophenyl octyl ether (NPOE), dithranol, $t$-3-indoleacrylic acid (IAA), 2,5-dihydroxybenzoic acid (DHB), sinapinic acid, and 2-(4-hydroxyphenylazo)-benzoic acid (HABA) and the cationization agent silver trifluoroacetate (AgTFA) were purchased from Aldrich Chemical Co. (Milwaukee, WI) and were used as received. Carbostyril was purchased from Kodak (Rochester, NY). The chloroform and acetone solvents were the Optima variety from Fisher Scientific (Fair Lawn, NJ).

Standard sample preparation. A procedure for sample preparation, derived from the Danis and Karr method [4], has been developed from which reproducibly good spectra are generated. For the analysis of PS, solutions of dithranol $\left(2 \times 10^{-1} \mathrm{M}\right)$, AgTFA $\left(4.5 \times 10^{-3} \mathrm{M}\right)$, and PS $\left(1 \times 10^{-4} \mathrm{M}\right.$ for $\left.10 \mathrm{ku}\right)$ were prepared in $\mathrm{CHCl}_{3}$. Fresh solutions of matrix and cationization agents were prepared daily (AgTFA was observed to fall out of solution and become cloudy and grey within few hours of preparation). The solutions were combined in a 5:1:1 volume ratio of matrix to polymer to cationization agent. A fine-tipped syringe that contained $1 \mu \mathrm{L}$ of the mixture was dragged across the sample plate (total deposition area approximately $5 \mathrm{~mm}^{2}$ ), which allowed the solvent to evaporate slowly. The resulting total sample load was $1.6 \times 10^{-7} \mathrm{~mol}$ of dithranol, $1.4 \times$ $10^{-11} \mathrm{~mol}$ of 10-ku PS, and $6.5 \times 10^{-10} \mathrm{~mol}$ of AgTFA, with a mole ratio of $11,000: 1: 45$. Samples that contained other polymers were prepared via the same procedure. For the analysis of PEO and PMMA, AgTFA 
was omitted from the preparation. Different matrices can be substituted for dithranol. IAA and DHB were not soluble in $\mathrm{CHCl}_{3}$; therefore, acetone was used as the solvent for polymer, matrix, and cationization agent. Acetone evaporates much more rapidly and may affect the physical properties of the resulting solid matrix.

Time-of-flight matrix-assisted laser desorption mass spectrometry. The instrumentation primarily used was a Kratos Kompact II MALDI-MS (Kratos Analytical, Ramsey, NJ), a time-of-flight (TOF) benchtop model designed for routine analysis of biomolecules. The Kratos instrument employs a $\mathrm{N}_{2}$ laser with a pulse length of 3 ns (as measured with a fast response diode). The beam spot is elliptical with a $0.4-\mathrm{mm}$ major axis and a $0.2-\mathrm{mm}$ minor axis (as measured by using facsimile paper at an angle $70 \%$ off normal). The laser rasters once across the sample in the $x$ direction, at a nonalterable $y$ point. Alternatively, the laser can be held at a specific point in the $x$ direction, especially when a "sweet spot" (site of high ion intensity) is observed. Normally, 100 laser shots are pulsed and the resulting spectra are summed. The extraction voltage in the Kratos instrument can be set at either 5 or $20 \mathrm{kV}$, with no postacceleration capabilities. The analyzer can be operated in either the linear or reflectron modes with flight paths of 1 or $2 \mathrm{~m}$. The ions are detected by a large area flat diode electron multiplier that uses a $300-\mathrm{MHz}$ direct current 8-bit "flash" analog-to-digital converter.

A few samples were analyzed by using a Perseptive Biosystems/Vestec LaserTec BenchTop Reflector II MALDI-MS (Framingham, MA). This system utilizes a $\mathrm{N}_{2}$ laser with 3-ns-wide pulses and a $10-30-\mu \mathrm{m}$ beam diameter. The ions are extracted with a variable voltage up to $30 \mathrm{kV}$ and analyzed in either linear $(1.3-\mathrm{m})$ or reflectron $(2.6-\mathrm{m})$ TOF modes. An electron multiplier is used for detection and the signal is recorded by a Tektronix Model 520 (Tektronix, Inc., Beaverton, OR) $500-\mathrm{MHz}$ digitizing oscilloscope. The components of this instrument suggest that it is more powerful for the characterization of polymers. The higher available extraction energy and the option to gate low mass ions may allow for increased detection sensitivity of the high mass molecules. Also, a video monitor is provided to view samples. Through control of laser beam position, critical regions of the sample can be probed. As a result, ion signal can be increased through easier identification of the location of "sweet spots."

Mass calibration is based on protein standards: Insulin (5734.6 u) was used to calibrate low molecular weight polymers and bovine serum albumin $(66,431 \mathrm{u})$ for high molecular weight polymers. A calibration constant was determined from the fit of several known masses (high and low masses) in the mass spectrum of the protein standard through the equation $m=k(t-$ $\left.t_{0}\right)^{2}$, where $m$ is mass, $k$ is the calibration constant, and $\left(t-t_{0}\right)$ is the time-of-flight of the ion through the analyzer. The appropriate calibration constant was applied to the mass spectra obtained during analysis of polymers. Fine tuning of the calibration was performed by identification of known peaks in the polymer mass spectrum (matrix peaks and alkali metals) and incorporation of their exact masses in the calibration fit.

Time-of-flight secondary ionization mass spectrometry. The TOF-SIMS spectra were acquired by using the TOF-II reflectron instrument developed and located at the University of Münster [8]. Typical instrumental conditions during TOF-II spectrum acquisition were 1.2-pA primary $\mathrm{Ar}^{+}$current with a pulse width of $1 \mathrm{~ns}$ and a repetition rate of $150 \mu \mathrm{s} /$ cycle. The primary beam strikes a $0.25-\mathrm{mm}^{2}$ analysis area during a $200-\mathrm{s}$ acquisition period. The analysis conditions were maintained within the static regime; primary ion doses were approximately $3 \times 10^{11}$ ions $/ \mathrm{cm}^{2}$. A $10-\mathrm{keV}$ postacceleration was employed. Samples were prepared for TOF-SIMS analysis by deposition of $1 \mu \mathrm{L}$ of polymer solution in chloroform $(1 \mathrm{mg} / \mathrm{mL})$ on about $80 \mathrm{~mm}^{2}$ of an etched silver substrate (monolayer preparation). The samples that contained physical mixtures of polymers were prepared by combination of a 1:1 mole ratio of the polymers prior to dissolution in chloroform. Three samples of each of the physical mixtures were prepared and analyzed.

Gel permention shromatography. In most cases, a Waters 150-CV GPC (Waters Chromatography, Milford, MA) with PS standards (Showa Denko) was used for measurements of molecular weight and polydispersity (PD). The instrument is operated with Ultrastyragel columns of $100-, 500-, 10^{3}-, 10^{4}-$, and $10^{5}-\AA$ porosities in tetrahydrofuran. Because GPC is a relative method to measure molecular weight, the determined values are accurate only for the polymer with which it has been calibrated. Therefore, the molar masses as determined by GPC for PI and PBD were normalized by using correction factors (1.35 and 1.75, respectively [9]). No correction factors have been used to report the molar mass values for PE, PEO, and PDMS.

\section{Results and Discussion}

\section{Investigation of Sample Preparation Procedure}

Matrix choice for polystyrene. The analysis of PS by MALDI-MS has been documented by four research groups. Table 1 shows the diverse approaches toward sample preparation taken by each group. An extensive investigation was done by the Hillenkamp group [3], who used a liquid matrix with the addition of AgTFA for Ag cationization. Most recently, Danis and coworkers [5] showed good results for PS by using the silver metal salt of IAA as a matrix. The feasibility of a solid matrix use was demonstrated by other groups 
Table 1. Approaches to sample preparation for polystyrene"

\begin{tabular}{|c|c|c|c|c|c|c|c|}
\hline \multirow[b]{2}{*}{ Reference } & \multirow[b]{2}{*}{ Matrix } & \multirow[b]{2}{*}{ Cation } & \multirow[b]{2}{*}{ Solvent } & \multicolumn{3}{|c|}{$\begin{array}{l}\text { Total moles deposited } \\
\text { (based on } 10 \mathrm{ku} \text { PS) }\end{array}$} & \multirow{2}{*}{$\begin{array}{c}\text { Molecular } \\
\text { weight } \\
\text { (ku) } \\
\text { analyzed }\end{array}$} \\
\hline & & & & Polymer & Matrix & Cation & \\
\hline Bahr & NPOE & AgTFA & Not necessary & $5 \times 10^{-11}$ & $4.1 \times 10^{-9}$ & $2.26 \times 10^{-9}$ & 1.3 \\
\hline \multirow[t]{8}{*}{ et al. [3] } & & & & & & & 1.7 \\
\hline & & & & & & & 5 \\
\hline & & & & & & & 7 \\
\hline & & & & & & & 10 \\
\hline & & & & & & & 20 \\
\hline & & & & & & & 32.6 \\
\hline & & & & & & & 46 \\
\hline & & & & & & & 70 \\
\hline Danis and & IAA & AgIAA & NI & $\mathrm{NI}$ & N! & NI & 11.6 \\
\hline Karr [5] & & & & & & & 120 \\
\hline Juhasz [10] & Dithranol & AgTFA & THF & $5 \times 10^{-11}$ & $2.2 \times 10^{-7}$ & NI & 9.2 \\
\hline $\begin{array}{l}\text { Kahr and } \\
\text { Wilkins [6] }\end{array}$ & DHB & $\mathrm{AgNO}_{3}$ & $\mathrm{NI}$ & NI & $\mathrm{NI}$ & $\mathrm{NI}$ & 8 \\
\hline \multirow[t]{7}{*}{ This paper } & Dithranol & AgTFA & $\mathrm{CHCl}_{3}$ & $1.4 \times 10^{-11}$ & $1.6 \times 10^{-7}$ & $6.5 \times 10^{-10}$ & 1 \\
\hline & & & & & & & 2.5 \\
\hline & & & & & & & 5 \\
\hline & & & & & & & 7.5 \\
\hline & & & & & & & 9 \\
\hline & & & & & & & 15 \\
\hline & & & & & & & 40 \\
\hline
\end{tabular}

${ }^{\mathrm{N}} \mathrm{NI}=$ not indicated

through a low mass example $[6,10]$. However, the exact sample preparation procedure for solid matrix use is not explained fully by any of the groups. Furthermore, it is not evident whether the addition of a cationization agent is necessary or why the particular agent was chosen, when used. Clearly, the optimal sample preparation method of PS for MALDI-MS has not been determined yet.

To choose a matrix, the first requirement to fulfill is that the matrix absorb at the wavelength of the laser to prevent extensive fragmentation of the polymer. Second, the matrix and analyte should have good miscibility in the condensed phase to reduce intermolecular interactions. Further criteria are difficult to identify because little is known about the role the matrix plays in the desorption and ionization of the analyte, particularly for polymers. A liquid matrix may be a good matrix choice because of its homogeneous nature and refreshable surface. One advantage of a solid matrix, though, is that it is easier to control during sample preparation and deposition. For biomolecules, the matrix is thought to play a role in ionization and to facilitate proton abduction. However, most polymer chains have been cationized by alkali metals or $\mathrm{Ag}$; ion formation does not appear to be a direct result of matrix type. Therefore, matrices that absorb in the UV region and are soluble in organic solvents were selected for this study (Table 2); they were chosen from a list of aromatic acid or alcohol matrices that are used routinely for biomolecule analysis.

As a starting point in development of a protocol for the analysis of PS, some of the approaches taken by the above-mentioned four groups were replicated to the extent possible. Use of the liquid NPOE matrix and AgTFA cationizing agent enabled distributions of oligomer chains to be obtained for 1- and 5-ku PS by using the Kratos TOF-MALDI-MS. Use of AgTFA as a cationization agent for PS, DHB, and IAA matrices was tried also. Very low signal in the mass range around 5 $\mathrm{ku}$ was obtained for the 5-ku PS in IAA, and no signal was obtained with the DHB matrix. The use of dithranol as a matrix with AgTFA gave by far the best results: PS up to a molecular weight of $40 \mathrm{ku}$ successfully was analyzed. In comparison to the preparation with NPOE matrix, signal could be obtained by using $50 \%$ lower laser power, and the intensity had over twice the signal-to-background ratio (S/B; Figure 1). HABA, sinapinic acid, and carbostyril also were employed as matrices with AgTFA cation, but no molecular signals were observed (Table 3 ).

The inefficacy of DHB and IAA as matrices in this investigation does not discredit the original reports: It serves to highlight the difficulties of development of a reproducible sample preparation and also emphasizes the success of dithranol as a matrix. Furthermore, difficulties were heightened due to the inability to 
Table 2. Structures of common matrices used in this study

\begin{tabular}{|c|c|c|}
\hline Matrix & Structure & Molecular weight (u) \\
\hline $\begin{array}{l}\text { 2-Nitrophenyl octyl ether } \\
\text { (NPOE) }\end{array}$ & & 251.33 \\
\hline Dithranol & & 226.23 \\
\hline $\begin{array}{l}\text { trans-3-Indoleacrylic acid } \\
\text { (IAA) }\end{array}$ & & 187.2 \\
\hline $\begin{array}{l}\text { 2,5-Dihydraxybenzoic acid } \\
\text { (DHB) }\end{array}$ & & 154.12 \\
\hline Sinapinic acid & & 224.21 \\
\hline Carbostyril 124 & & 174.20 \\
\hline $\begin{array}{l}\text { 2-(4-Hydroxyphenylazo)- } \\
\text { benzoic acid } \\
\text { (HABA) }\end{array}$ & & 242.23 \\
\hline
\end{tabular}

position the laser freely on the sample, because it is possible that the most intense ion signal comes from localized regions of the sample. Thus, even for a sample prepared with optimal conditions, the instrument may fail to produce molecular ions from the analyte.

Other polymers and matrices. The sample preparation method developed for PS was applied to different polymers in the same hydrocarbon class. Molecular ions were observed only for 1-ku samples of PI and PBD in dithranol matrix with AgTFA (Table 3). No molecular ions were observed for PE with the same preparation. One problem may be combination of the appropriate amount of polymer with the matrix. Whereas PS is a solid material, PBD, PI, and PE are gums (due to lower glass transition temperatures) and may require different mole quantities of polymer to matrix. Furthermore, the structure of these materials makes them inherently difficult to ionize, similar to PS; PBD, PI, and PE contain even fewer sites of unsaturation than PS. Further investigation into the utility of different matrices is necessary.

The sample preparation method developed for PS also was applied to a polar polymer (PEO). However, no added cationization agent was necessary. Molecular ions were observed for 5- and 11-ku PEO in dithranol with contaminant $\mathrm{Na}$, probably from the $\mathrm{CHCl}_{3}$, serving to cationize the oligomers. The analysis of PEO in a matrix of DHB gave comparable results. Note that for PS, only dithranol is a good matrix, but both DHB and dithranol are good matrices for PEO. Perhaps PS, with its phenyl functionality, is highly miscible in dithranol because of the largely aromatic structure of the matrix. For PEO, solubility in DHB or dithranol may be high because of the alcohol functionality of the matrices.

The sample preparation method developed for PS failed when applied to PMMA; no molecular ions could be obtained when dithranol was used as a matrix. Good molecular ion signals were obtained for PMMA in a matrix of IAA, however. Here also, no added cationization agent is necessary. Because at least low signals were obtained for PMMA in a matrix of $\mathrm{DHB}$, the acid functionality of the matrices (IAA or $\mathrm{DHB})$ is thought to promote miscibility of PMMA and matrix.

The sample preparation method also was successful for the analysis of 5-ku polydimethylsiloxane (PDMS) in a matrix of dithranol with AgTFA. Other matrices also may be useful, but none was evaluated in this study for PDMS. 


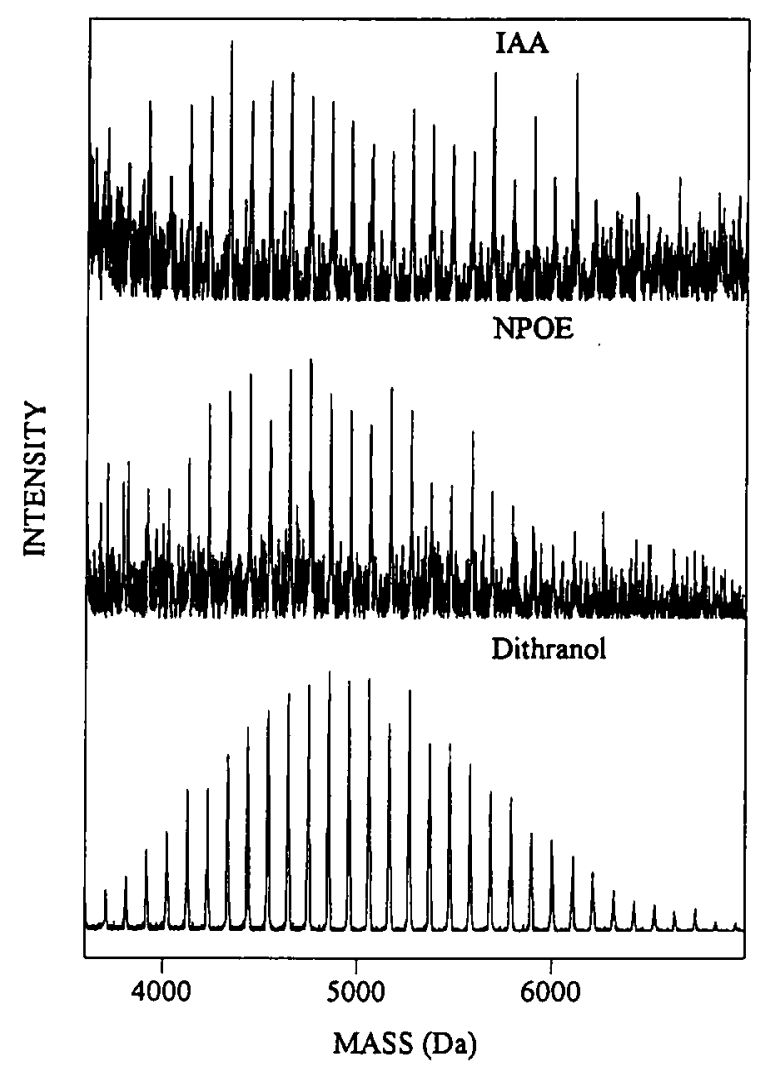

Figure 1. Comparison of TOF-MALDI-MS spectra obtained for 5-ku PS by using different matrices: IAA (top), NPOE (middle), and dithranol (bottom). No oligomer signals were obtained for matrices HABA, sinapinic acid, and carbostyril. AgTFA was used as a cationization agent in all sample preparations.

Direct laser desorption of polymers. The necessity of matrix and cationization agent was investigated by laser desorption experiments. No matrix or cationization agent is necessary to obtain molecular ion signals for 1-ku PS, which is consistent with literature reports that UV laser desorption can be used for polymers under $2 \mathrm{ku}[12,13]$. Furthermore, no molecular ion signals were observed by direct laser desorption of 5-ku PS, PEO, or PMMA. With the addition of cation (still no matrix), however, molecular ions were observed for a 5-ku PS sample, albeit of low intensity. The use of matrix in high concentrations $\left(10^{3}-10^{4}\right.$ mole excess) was verified to facilitate desorption and ionization of polymers, as evidenced by the increased $\mathrm{S} / \mathrm{B}$ for the 5-ku samples and the capability to analyze even higher molecular weight samples, despite dilution of the analyte.

Cationization agents. The utility of cationization agents was explored also (Table 4). In the analysis of polar materials, the addition of a cationization agent is not always necessary. Frequently, alkali metals ( $\mathrm{Li}, \mathrm{Na}, \mathrm{K}$ ) inherent to the matrix or solvent used in sample preparation serve to cationize the molecular ions (PMMA and PEO are good examples). The ion yield sometimes is increased by the intentional addition of alkali salts.
In this investigation, the alkali metals were not effective as cationization agents for the nonpolar PS polymer. No molecular ions were generated for PS with alkali metal adducts in dithranol matrix. Furthermore, the addition of $\mathrm{NaCl}$ and $\mathrm{KCl}$ to the sample did not yield molecular ions. Because these salts are not soluble in the organic material used for sample preparation, they may not be dispersed effectively throughout the matrix. More likely, the absence of heteroatoms $(\mathrm{N}$, $\mathrm{O}$ ) in the polymer prevents adduct formation. Silver, however, proves to be an effective cationization agent for PS and has been employed in laser desorption-mass spectrometry (LDMS) and SIMS experiments to aid in cationization of PS molecular ions [14, 15]. In our MALDI experiments, the addition of AgTFA (soluble in organic solvents) to the matrix allows PS molecular ions to be generated with $\mathrm{Ag}$ adducts. Furthermore, AgTFA was found to be an effective cationization agent for the hydrocarbon polymers PI and PBD, but not for PE. This suggests that the affinity of $\mathrm{Ag}$ for a double bond promotes adduct formation. The affinity is investigated by analysis of polymers of different structures. Upon the addition of AgTFA to a matrix that included PEO, only molecular ions with $\mathrm{Na}$ adducts ( $\mathrm{Na}$ from solvent) are observed. $\mathrm{Ag}$ does not have an affinity for this completely saturated polymer with $\mathrm{O}$ moieties. In comparison, when AgTFA is added to a matrix including PMMA, both Ag and $\mathrm{Na}$ cationized molecular ions form. In this case, the Ag may form an adduct with the ester functionality. Finally, $\mathrm{Ag}$ also is observed to form adducts with PDMS to form molecular ions. PDMS, with its $\mathrm{Si}-\mathrm{O}$ backbone, also has been shown to be cationized with $\mathrm{Na}$ and $\mathrm{K}$ [16]. The mechanism for the formation of Ag-cationized molecular ions is of much interest. The data provided in this study, however, do not provide information to indicate whether the ions are formed as neutral species and ionize in the process of desorption or if the adduct is formed in the gas phase after desorption.

\section{Evaluation of Instrumental Parameters}

Typical results. The characteristics of a typical MALDI spectrum are illustrated in Figure 2 via the spectrum of a 5-ku PS sample prepared by using dithranol matrix. In the mass range above $3 \mathrm{ku}$, the signals represent intact oligomer ions. The mass at which the signals arise represents the sum of the mass of the terminating groups on the polymer, the mass of a specific number of repeat units, and the mass of a silver atom, which aids in cationization. The signals are spaced by the mass of the repeat unit (104 u, for PS). In contrast to a TOF-SIMS spectrum, no polymer fragment ions are observed. The only low mass signals present are due to the matrix, the cationization agent, and alkali metals.

Frequently, clusters of the molecular ions (dimers, trimers) and multiply charged molecular ions (doubly, triply) are observed, particularly in higher molecular 
Table 3. Molecular weights of polymers analyzed in different matrices

\begin{tabular}{|c|c|c|c|c|c|c|}
\hline \multirow[b]{2}{*}{ Matrix } & \multirow[b]{2}{*}{ Solvent } & \multicolumn{5}{|c|}{ Molcular weights of polymers analyzed ${ }^{\mathrm{a}}(\mathrm{ku})$} \\
\hline & & $\begin{array}{c}\mathrm{PS} \\
\mathrm{W} / \mathrm{Ag}\end{array}$ & $\begin{array}{c}\mathrm{PI} \\
w / \mathrm{Ag}\end{array}$ & PEO & PMMA & Other \\
\hline \multirow[t]{3}{*}{$\overline{\mathrm{NPOE}}$} & None & 1 & NA & NA & NA & NA \\
\hline & necessary & 2.5 & & & & \\
\hline & (liquid matrix) & 5 & & & & \\
\hline \multirow[t]{7}{*}{ Dithranol } & Chloroform & 1 & 1 & & & PBD w/Ag: $1 \mathrm{ku}$ \\
\hline & & 2.5 & & & & \\
\hline & & $5^{t}$ & & $5^{\dagger}$ & & PDMS w/Ag: $5 \mathrm{ku}$ \\
\hline & & 7.5 & & $11^{\dagger}$ & NMI & PE w/Ag: $7.5 \mathrm{ku}$ (NMI) \\
\hline & & $9^{t}$ & & & & \\
\hline & & $15^{\dagger}$ & & & & \\
\hline & & $40^{*}$ & & & & \\
\hline \multirow[t]{6}{*}{ IAA } & Acetone & 5 & 1 & & & \\
\hline & & & 5 & & $5^{\dagger}$ & \\
\hline & & & & NA & 10 & NA \\
\hline & & & & & $19^{*}$ & \\
\hline & & & & & $28^{*}$ & \\
\hline & & & & & $49^{*}$ & \\
\hline \multirow[t]{2}{*}{$\mathrm{DHB}$} & Acetone & NMI & NA & 1 & 1 & NA \\
\hline & & & & 5 & 5 & \\
\hline Sinapinic acid & Acetone & NMI & NA & NA & NA & NA \\
\hline Carbostyril & Acetone & NMI & NA & NA & NA & NA \\
\hline HABA & Acetone & NMI & NA & NA & NA & NA \\
\hline
\end{tabular}

Note: All spectra were acquired on the Kratos instrument unless otherwise indicated. An asterisk ( $\left.{ }^{\star}\right)$ indicates a spectrum acquired on the Vestec instrument; a dagger ( $\dagger$ ) indicates spectra acquired on both Kratos and Vestec instruments

a $\mathrm{NA}=$ not attempted; $\mathrm{NMI}=$ no molecular ions (sample was prepared and analyzed, but preparation failed to yield polymer signalsl; $\mathrm{w} / \mathrm{Ag}=\mathrm{AgTFA}$ was used in the sample preparation and oligomers are cationized by $\mathrm{Ag}$.

weight polymers. Their presence and increasing significance with molecular weight of polymer could be related to polymer chain length. The longer polymer chains may become associated more easily before laser desorption and remain so afterwards, which creates molecular clusters. Longer polymer chains also have more available sites for ionization, which increases their probability of becoming multiply charged ions. Contributions from the signals of clusters and multiply charged species may be problematic in higher molecular weight samples by skewing the signal for the distribution of singly charged molecular ions.

High molecular weight polymers. The highest molecular weight polymer from which molecular ion signal could be obtained in this study was a $49-\mathrm{ku}$ sample of PMMA in I $\Lambda \Lambda$ (Figure 3). The higher molecular weight polymers are more difficult to analyze as evidenced by the low signal intensity of the molecular ions. Both the sample preparation procedures and the instrumental parameters contribute to the lower sensitivity for higher molecular weights. The higher molar mass materials tend to have broader polydispersities (greater total number of chain lengths in the distribution). As a consequence, fewer total moles of each of the oligomers are loaded onto the sample. Furthermore, the higher molar mass polymers have longer chains. Therefore, even though the matrix:polymer mole ratio may remain the same (compared to easily analyzed low molecular weight polymer), the matrix may not be as effective in surrounding and solvating the polymer. Increasing the concentration of matrix may be counterproductive because it reduces the already limited amount of polymer molecular ions. Finally, the higher molecular weight ions are more massive and require a higher extraction and/or postacceleration to maintain detector sensitivity [17]. The Kratos instrument may be limited in that it has a maximum extraction energy of $20 \mathrm{keV}$. The extraction-postacceleration energy combination of other MALDI instrumentation is often in the $30-35-\mathrm{keV}$ range.

Analyzer mode. The TOF-MALDI-MS instruments are capable of analysis in both linear and reflectron modes. Typically, in the analysis of biomolecules, samples under $10 \mathrm{ku}$ are analyzed in the reflectron mode to achieve higher mass resolution. The linear mode, although lower in mass resolution, is used for samples above $10 \mathrm{ku}$. The linear analyzer is expected to provide higher transmission for higher mass species due to its shorter flight path and the absence of the reflectron (refocusing) region. 
Table 4. Metals that serve to cationize polymer chains when added to the matrix

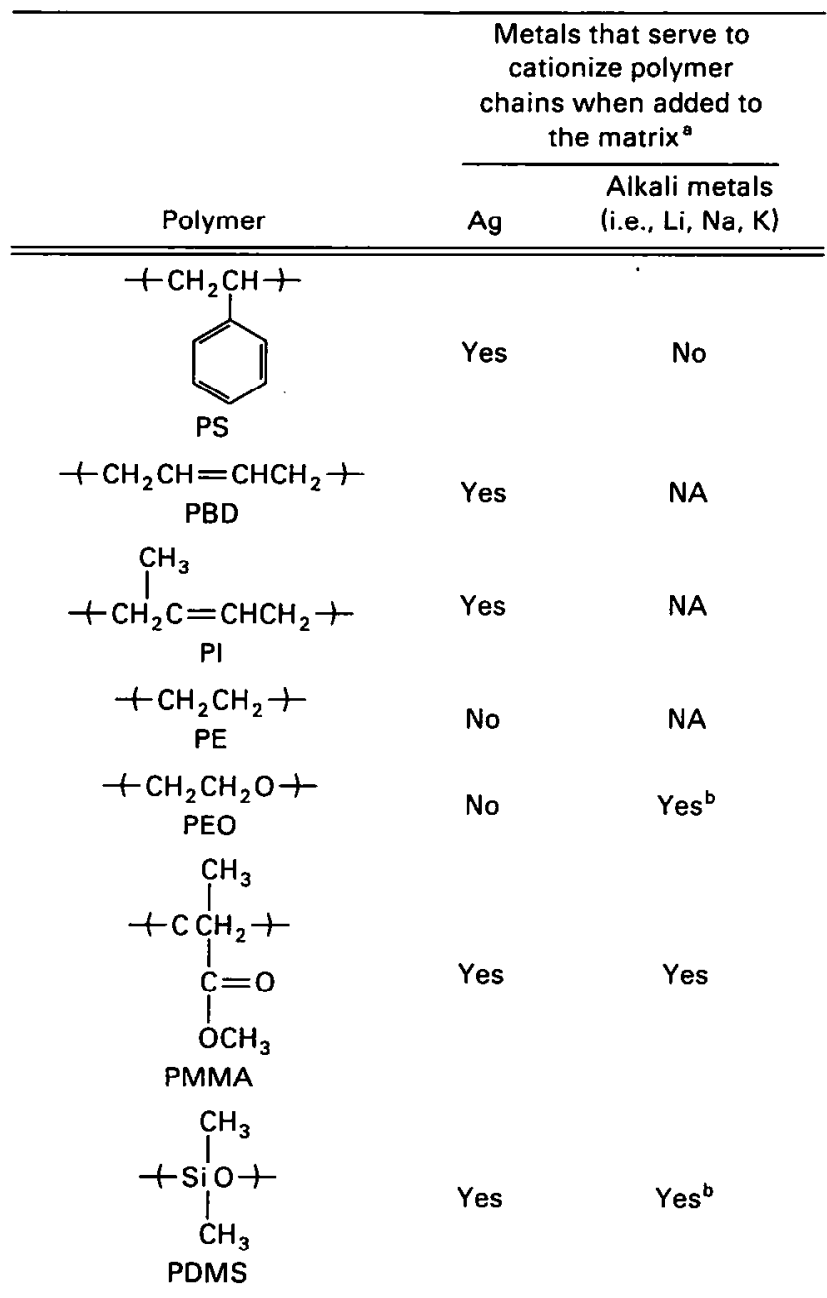

- $N A=$ not attempted.

As determined from published reports

The capabilities of the linear and reflectron modes of the Kratos instrument were compared in this investigation for polymeric samples (Table 5). The reflectron mode of the analyzer allows the detection of the individual oligomers of 1-ku PS. At this molecular weight, the resolution ( $m / \Delta m$ ) was calculated to be 210 , with a full width at half maximum (FWHM) of an oligomer signal approximately $5 \mathrm{u}$. As a consequence, isotopic species within each oligomer cannot be identified. The linear mode offers no significant advantages or disadvantages for the analysis of PS at this molecular weight; although the resolution (FWHM $=15 \mathrm{u}, m / \Delta m=100$ ) is lower, individual oligomers still can be resolved easily. Furthermore, both linear and reflectron modes are comparable in sensitivity. For the analysis of 7-ku PS, better results were obtained consistently by using the reflectron mode. Here, the FWHM of the oligomer signal degrades to approximately $35 \mathrm{u}(m / \Delta m=190)$, but the signal [based on signal-to-background (S/B) calculations] is still strong. In comparison, the signal intensity that results from the linear mode is poor, in
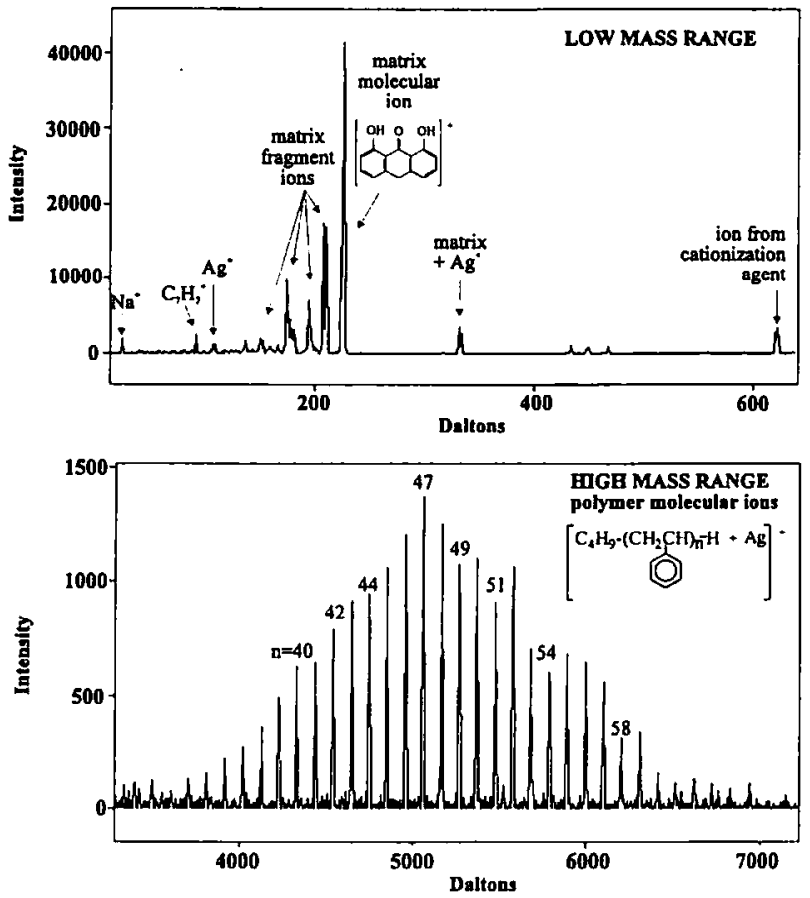

Figure 2. Typical TOF-MALDI-MS spectrum: polystyrene in dithranol matrix with AgTFA cationization agent.

addition to lower resolution (FWHM $=60 \mathrm{u}, m / \Delta m=$ 115; Figure 4). The reflectron mode was employed also for the analysis of 15-ku PS. Although the signal intensity is weak for this high molecular weight sample, the FWHM of the oligomer signals remains at approxi-

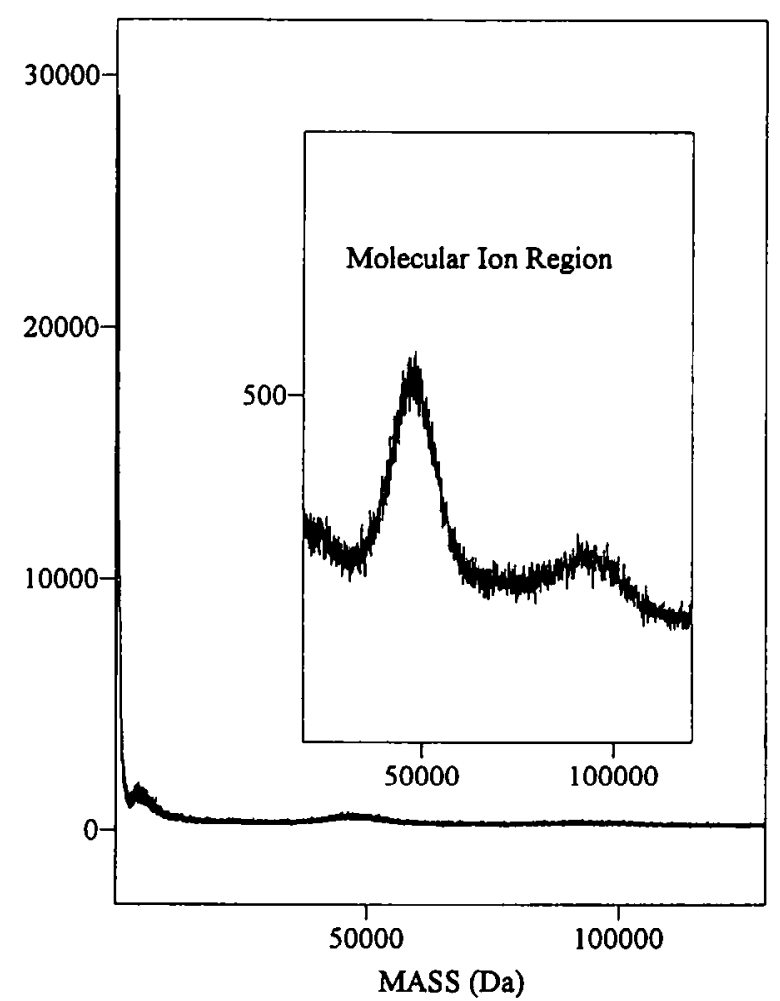

Figure 3. TOF-MALDI-MS spectrum of 49-ku PMMA in IAA matrix. 
Table 5. Comparison of reflectron and linear time-of-flight analyzer modes

\begin{tabular}{|c|c|c|c|c|c|c|}
\hline \multirow[b]{2}{*}{$\begin{array}{l}\text { MW } \\
\text { (ku) }\end{array}$} & \multicolumn{3}{|c|}{ Reflectron } & \multicolumn{3}{|c|}{ Linear } \\
\hline & $\begin{array}{c}\text { Resolution } \\
(\mathrm{m} / \Delta \mathrm{m})\end{array}$ & $\begin{array}{c}\text { FWHM } \\
\text { (u) }\end{array}$ & $\begin{array}{l}\text { Signal-to- } \\
\text { background }\end{array}$ & $\begin{array}{l}\text { Resolution } \\
(m / \Delta m)\end{array}$ & $\begin{array}{c}\text { FWHM } \\
\text { (u) }\end{array}$ & $\begin{array}{l}\text { Signal-to- } \\
\text { background }\end{array}$ \\
\hline 1 & 210 & 5 & 270 & 100 & 15 & 110 \\
\hline 7 & 190 & 35 & 220 & 115 & 60 & 3 \\
\hline 15 & 434 & 35 & 3 & - & - & $<3$ \\
\hline 7 (Vestec) & NA & NA & NA & 110 & 45 & 6 \\
\hline
\end{tabular}

${ }^{\mathrm{a}} \mathrm{NA}=$ not attempted.

mately $35 \mathrm{u}$ (Figure 5). Very little signal could be obtained in the linear mode for this molecular weight polymer. The decreased sensitivity of the linear mode is uncharacteristic and cannot be explained. The advantages of the reflectron mode, however, are not expected to persist for higher molecular weight samples. The weak signal that was obtained for the $15-\mathrm{ku}$ sample could not be increased by varying sample preparation nor could higher molecular weight samples be analyzed in the reflectron mode.

Polymer samples also were analyzed in the linear mode of the Vestec instrument. Here the flight tube is longer, but the acceleration energy is higher. As a result, signal intensity for 7-ku PS is stronger for the Vestec instrument than for the Kratos instrument, in the linear mode. The resolution is approximately the same for the Vestec (FWHM $=45 \mathrm{u}, m / \Delta m=110$ ).

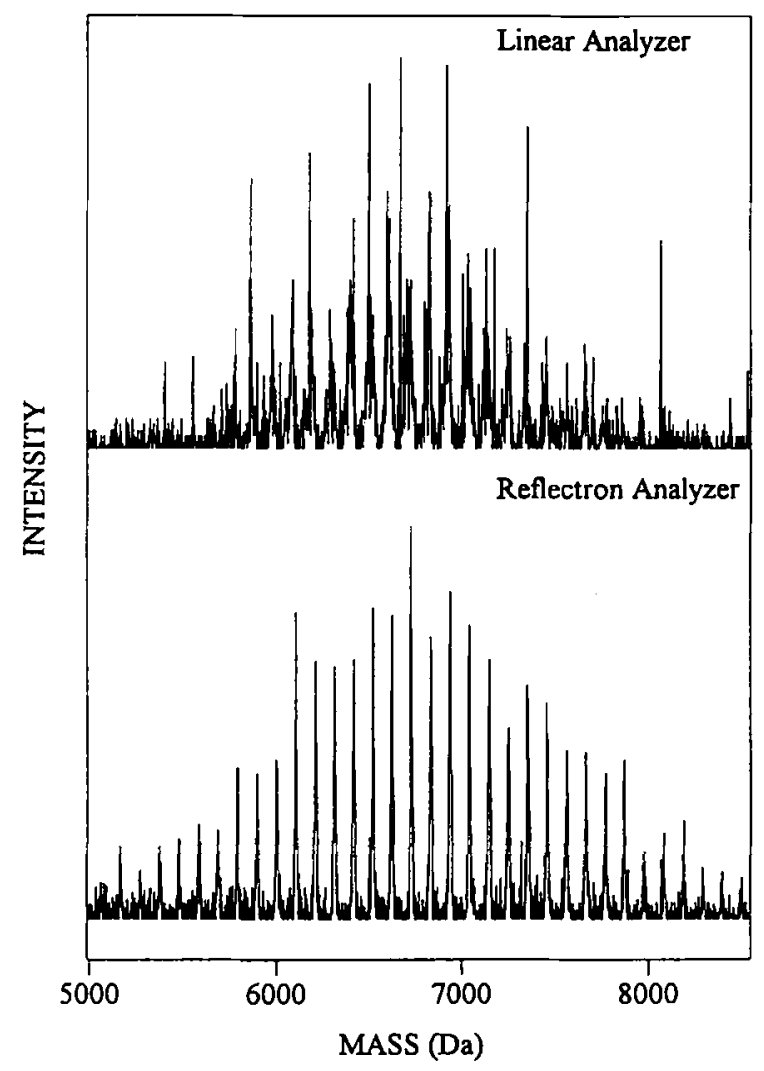

Figure 4. Comparison of linear and reflectron modes for the analysis of the same sample of 7-ku PS.
For polymer characterization, the resolution of the instruments is adequate for identification of repeat units. The end groups of polymers under $15 \mathrm{ku}$ may be verified also if their structure is known a priori. The FWHM of the molecular ions is too broad to allow structural elucidation of unknown end groups.

Laser intensity. The effect of laser intensity on TOFMALDI-MS polymer spectra was investigated. TOFMALDI-MS measurements were made on three identical preparations of each polymer; $M_{\mathrm{n}}$ and S/B values were calculated from the areas and masses of the intact oligomer signals, and were averaged for the three samples. The calculated $M_{\mathrm{n}}$ values were corrected by subtracting the mass of the cationization agent (e.g., $108 \mathrm{u}$ was subtracted from the $M_{\mathrm{n}}$ value for $\mathrm{Ag}$ cationized oligomers). "Optimal" laser intensity was designated as the power at which the strongest molecular ions signals could be obtained. As observed for

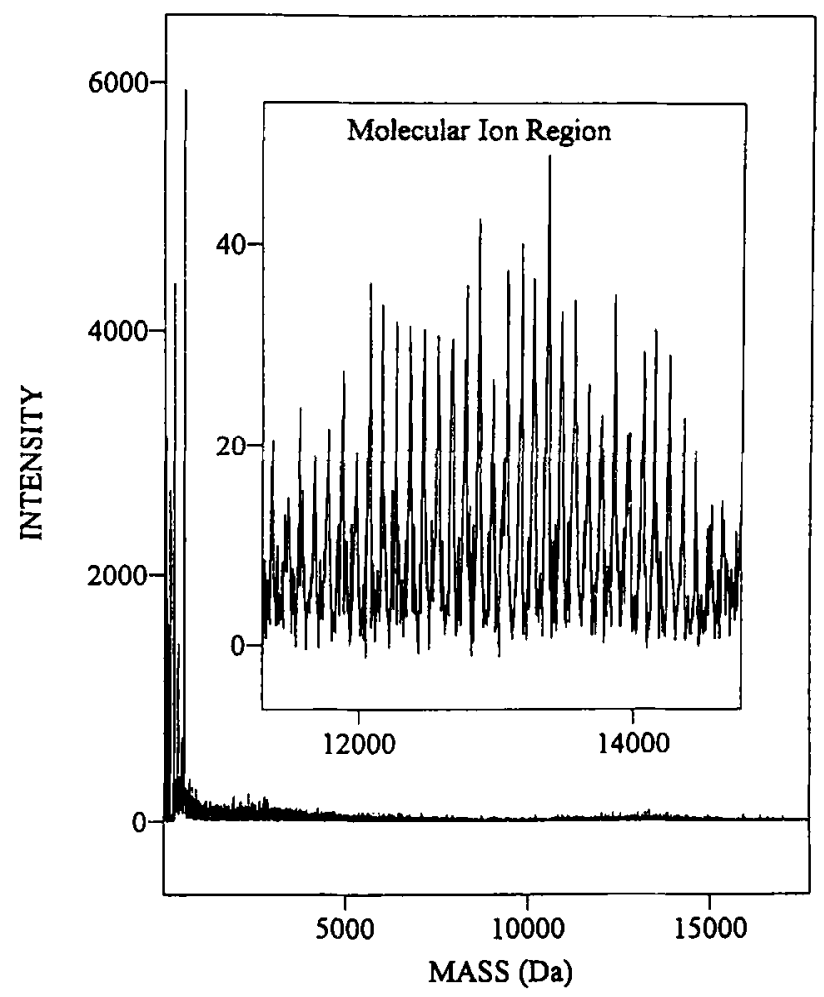

Figure 5. TOF-MALDI-MS spectrum of 15-ku PS obtained in reflectron mode. 
7-ku PS, the laser power could be increased or decreased by approximately $20 \%$ from this point and still yield molecular ion signals. However, the molecular ion signal intensity and the $S / B$ were poorer than at "optimal" irradiance. The calculated molecular weight values were consistent for all laser powers ( $6805 \pm 55$ u). Nonetheless, high and low mass oligomer signals are absent from the distribution when the power is not "optimal."

In contrast, for 1-ku PS, variations in laser intensity influenced the resulting distribution of molecular ion signals (Figure 6). At $100 \%$ power the laser pulse energy is $200 \mu \mathrm{J} \pm 10 \%$ with an average power of 2 $\mathrm{mW}$ at $20 \mathrm{~Hz}$. At a lower- "optimal"- -laser intensity, a distribution of molecular ions was generated, with a calculated $M_{n}=1132 \pm 29$ u. At $20 \%$ lower than optimal intensity-the "threshold" intensity-the molecular ion signals are not as intense and the $\mathrm{S} / \mathrm{B}$ is lower. No molecular ion signals were observed below threshold laser intensities. However, the distribution obtained at threshold is much more Gaussian and representative of an expected oligomer distribution. Furthermore, the relative intensities of the higher mass oligomers are greater, and the calculated $M_{\mathrm{n}}$ is shifted to $1240 \pm 17 \mathrm{u}$. The GPC-determined molecular weight for this polymer, $1210 \pm 55 \mathrm{u}$, also agrees more closely with the spectrum acquired at the lower laser power. When the laser intensity is increased to $20 \%$ above optimal power, the distribution flattens. The $M_{n}$ value $(1182 \mathrm{u})$ calculated is at an intermediate value to the previous two, which suggests an enhancement of higher mass ion yields, but with a concomitant increase in fragmentation relative to the optimal irradiance. Overall, the laser power may have a greater affect on the intensities of lower molecular weight polymer because the percentage change in size and mass is more significant for the lower mass oligomers with successive degrees of polymerization.

Comparison of Time-of-Flight Matrix-Assisted Laser Desorption Ionization and Time-of-Flight Secondary Ionization Mass Spectrometry Measurements

Evaluation of molecular weight distributions. Molecular weight values were calculated for all polymers analyzed by TOF-MALDI-MS. The $M_{n}$ values determined by TOF-MALDI-MS are compared to those determined by TOF-SIMS (only selected lots were available) and GPC in Table 6 . The $M_{\mathrm{n}}$ values determined by TOFMALDI-MS are consistently higher than values determined by TOF-SIMS for all classes of polymers with molecular weights up to $8 \mathrm{ku}$. Above $8 \mathrm{ku}$, no signal was observed in the high mass range by TOF-SIMS, and therefore $M_{\mathrm{n}}$ could not be calculated from the intact oligomers. Furthermore, the values determined by TOF-SIMS may be skewed toward the low mass end because of decreasing ion yields of oligomers with increasing mass. For 1-ku PS, the disappearance cross

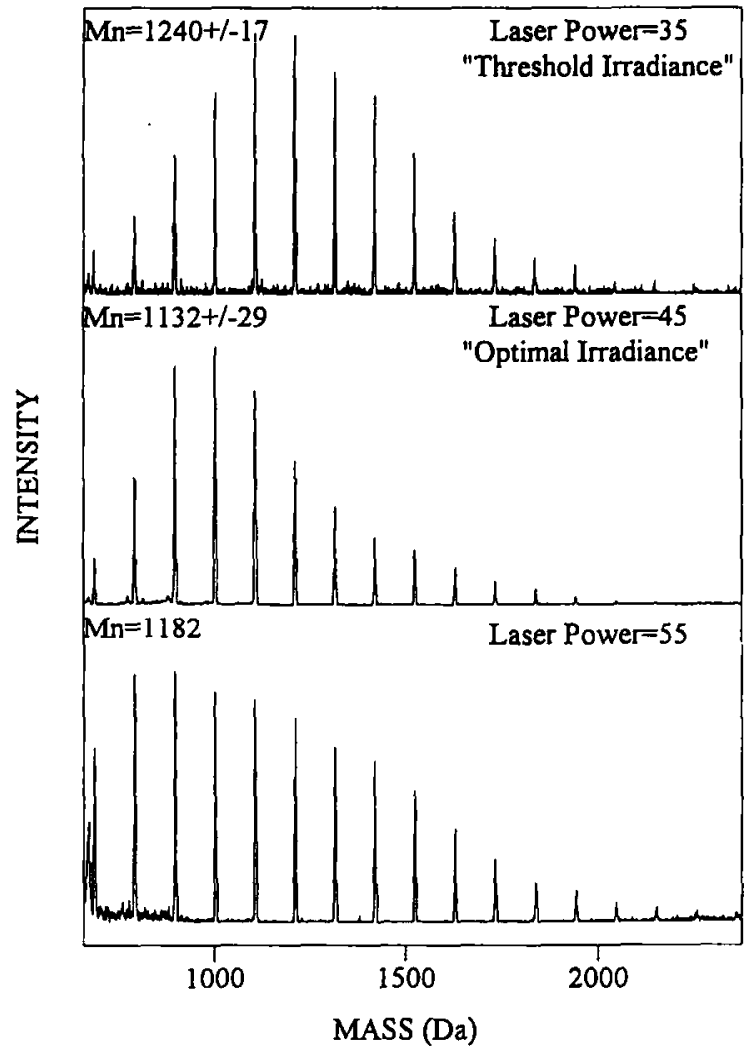

Figure 6. Changes in molecular weight distribution of 1-ku PS due to variations in laser power by TOF-MALDI-MS. GPC $M_{n}=$ 1210.

section (probability of molecular damage induced by the impact of a primary ion on a surface) was determined to increase by an order of magnitude over the molecular weight distribution [18]. Also, the transformation probability (likelihood that a molecule sputtered from the surface is emitted as a specific secondary ion species) of oligomers decreases with increasing mass. Together, these factors are detrimental to the calculation of accurate $M_{\mathrm{n}}$ values by TOF-SIMS. In comparison, for the TOF-MALDI-MS technique, ion yields of oligomers as a function of mass have not been investigated yet. In this study, one influence on the determination of $M_{n}$ by TOF-MALDI-MS is shown to be the laser power. Nonetheless, both TOF-MALDI-MS and TOF-SIMS $M_{\mathrm{n}}$ values agree well with those determined by GPC, given the low accuracy of GPC in the low mass range.

The accuracy of $M_{n}$ determinations also can be evaluated within each technique. The $M_{n}$ values of polymers that originate from the same "parent solution," but with different terminating groups, can be compared. The $M_{n}$ values should differ exactly by the mass of the terminating group. The shift in $M_{n}$ can be observed with all three techniques. However, the shift is more accurately characterized with mass spectrometry techniques, where the difference in $M_{n}$ values is found to deviate by $6 \%$ of the expected shift. With GPC, the shift in $M_{n}$ is found to vary by $16 \%$. 
Table 6. Comparison of molecular weight values determined by TOF-MALDI-MS, TOF-SIMS, and GPC

\begin{tabular}{|c|c|c|c|c|c|c|c|c|}
\hline \multirow{2}{*}{$\begin{array}{c}\text { Class } \\
\text { of } \\
\text { Polymer }\end{array}$} & \multirow{2}{*}{$\begin{array}{l}\text { MW } \\
\text { (ku) }\end{array}$} & \multirow{2}{*}{$\begin{array}{l}\text { Reference } \\
\text { name }\end{array}$} & \multicolumn{2}{|c|}{ TOF-MALDI-MS } & \multicolumn{2}{|c|}{ TOF-SIMS } & \multicolumn{2}{|c|}{ GPC } \\
\hline & & & $M_{n}$ & PD & $M_{n}$ & $P D$ & $M_{\mathrm{n}}$ & $P D$ \\
\hline \multirow[t]{18}{*}{$\overline{P S}$} & 1 & PS-H & 1130 & 1.05 & 971 & 1.07 & 930 & 1.13 \\
\hline & & & $1330^{\mathrm{KL}}$ & $1.03^{\mathrm{KL}}$ & & & & \\
\hline & & PS-405 & 1500 & 1.04 & 1414 & 1.04 & 1,490 & 1.07 \\
\hline & & PS-135 & 1200 & 1.04 & 975 & 1.04 & 1,200 & 1.10 \\
\hline & 2.5 & PS-H & 2450 & 1.03 & NA & NA & 2,010 & 1.07 \\
\hline & & PS-130 & 2420 & 1.03 & NA & NA & NA & NA \\
\hline & 5 & PS-H & 5200 & 1.01 & NA & NA & 4,340 & 1.06 \\
\hline & & & $5450^{\mathrm{VL}}$ & $1.02^{V L}$ & & & & \\
\hline & & PS-405 & 5530 & 1.01 & NA & NA & 4.760 & 1.05 \\
\hline & 7 & PS-H & 6810 & 1.01 & NMI & NMI & 7,140 & 1.05 \\
\hline & & & $6640^{\mathrm{KL}}$ & $1.01^{\mathrm{KL}}$ & & & & \\
\hline & & PS-135 & 6850 & 1.01 & 6415 & 1.01 & 7.220 & 1.05 \\
\hline & 9 & PS & 8990 & 1.01 & NA & NA & 9,000 & 1.03 \\
\hline & & & $9240^{\mathrm{VL}}$ & $1.01^{V L}$ & & & & \\
\hline & & PS-H & 8950 & 1.01 & NA & NA & 8,060 & 1.05 \\
\hline & 15 & PS-H & $14950^{\mathrm{VL}}$ & NC & NMI & NMI & 14,570 & 1.05 \\
\hline & & PS-135 & 13620 & 1.01 & NMI & NMI & 14,560 & 1.05 \\
\hline & 40 & PS-H & $51200^{\mathrm{VL}}$ & NC & NMI & NMI & 41,820 & 1.08 \\
\hline \multirow[t]{2}{*}{ PI } & 1 & $\mathrm{PI}-\mathrm{H}$ & 1170 & 1.04 & 891 & 1.05 & 970 & 1.12 \\
\hline & & PI-405 & 1510 & 1.03 & 1350 & 1.03 & 1,280 & 1.08 \\
\hline \multirow[t]{2}{*}{ PBD } & 1 & PBD-H & 1050 & 1.03 & 830 & 1.04 & 920 & 1.11 \\
\hline & & PBD-405 & 1440 & 1.02 & 1260 & 1.03 & 1,220 & 1.08 \\
\hline \multirow[t]{5}{*}{ PEO } & 1 & 73-PEO-43 & 1130 & 1.03 & NA & NA & 1,240 & 1.13 \\
\hline & 5 & 73-PEO-43 & 3790 & 1.01 & NA & NA & 5.140 & 1.10 \\
\hline & & & $4110^{V L}$ & $1.01^{\mathrm{VL}}$ & & & & \\
\hline & 11 & PEO & 8340 & 1.00 & NA & NA & 11,000 & NA \\
\hline & & & $8520^{\mathrm{VL}}$ & NC & & & & \\
\hline \multirow[t]{7}{*}{ PMMA } & 5 & PMMA-H & 5560 & 1.02 & NA & NA & 4,690 & 1.07 \\
\hline & & & $6730^{\mathrm{VL}}$ & $1.01^{\mathrm{VL}}$ & & & & \\
\hline & 19 & PMMA & $16510^{\mathrm{VL}}$ & NC & NA & NA & 19,000 & NA \\
\hline & & & $16090^{\mathrm{KL}}$ & NC & & & & \\
\hline & 28 & PMMA & $22900^{\mathrm{VL}}$ & NC & NA & NA & 28,000 & NA \\
\hline & 49 & PMMA & $46000^{\mathrm{Vl}}$ & NC & NA & NA & 49,000 & NA \\
\hline & & & $48100^{K L}$ & NC & & & & \\
\hline PDMS & 8 & PDMS-227 & 5050 & 1.02 & NA & NA & 7,600 & 1.06 \\
\hline
\end{tabular}

${ }^{a}$ All GPC values are versus polystyrene standards. All MALDI-MS spectra were obtained on the Kratos instrument in reflectron mode unless indicated by KL or VL: KL indicates MALDI-MS spectrum obtained on the Kratos instrument in the linear mode; VL indicates MALDI-MS spectrum obtained on the Vestec instrument in the linear mode. NA= not attempted; NMI= measured this sample and molecular ions not generated; $\mathrm{NC}=$ not calculated because of nonresolved oligomer signals.

The $M_{n}$ values determined by TOF-MALDI-MS and GPC were compared for different classes of polymers. For PS between 5 and $15 \mathrm{ku}$, the TOF-MALDI-MS $M_{n}$ varies up to $\pm 10 \%$ from the value determined by GPC. The TOF-MALDI-MS $M_{n}$ of $40-\mathrm{ku}$ PS is $22 \%$ higher than the GPC value, but the $S / B$ in this spectrum is quite low. Given the accuracy of the GPC $M_{n}$ of $\pm 5 \%$, some of the values determined by TOFMALDI-MS may be systematically high or low. Further investigation is necessary to determine the preci- sion of the TOF-MALDI-MS values. For PEO, PMMA, and PDMS (range in molecular weight 1-49 ku), the TOF-MALDI-MS values are lower by $10-20 \%$ compared to the GPC values. 5-ku PMMA is an exception; it has an $M_{n} 20 \%$ higher. The GPC-determined values may be superficially high because of differences in polarity and hydrodynamic volume of the polymers compared to the PS standards, which may allow them to elute from the column more quickly. In contrast, the TOF-MALDI-MS $M_{n}$ values for PI and PBD (1 ku) are 
15-25\% higher than GPC. The nonpolar PI and PBD are similar to PS, for which $M_{\mathrm{n}}$ values are also higher as determined by TOF-MALDI-MS.

In the mass range below $10 \mathrm{ku}$, the molecular weight distribution signals obtained by mass spectrometric techniques are clearly more informative than GPC. In addition to accurate $M_{\mathrm{n}}$ values, TOF-SIMS and TOFMALDI-MS allow structure elucidation through the resolved oligomer signals. Moreover, TOF-SIMS allows isotopic resolution of the oligomer signals. In the mass range above $10 \mathrm{ku}$, the capabilities of the techniques differ. TOF-SIMS is no longer useful for calculation of Mn from intact oligomer signals. GPC is not limited by molecular weight range and it is most accurate for PS because it is calibrated based on PS standards. Above $10 \mathrm{ku}$ with TOF-MALDI-MS, unresolved distributions of polymer chains are observed similar to GPC, but TOF-MALDI-MS is an absolute method to determine molecular weight. The limitation of the TOF-MALDIMS technique to determine molecular weight values remains in finding appropriate matrices for different classes of polymers and defining a standard set of instrumental parameters to obtain reproducible results.

Effect of endgroup on polymer ion yield. Molecular ion intensities may be influenced by desorption probability, extent of fragmentation and ionization, and detection probabilities of an oligomer. For a polymer chain of low molecular weight and with a narrow polydispersity (in this study $M_{\mathrm{n}}=1 \mathrm{ku}, \mathrm{PD}=1.10$ ), these factors are assumed to be constant. However, the presence of an end group alters the chemical composition of the molecules and can have a significant effect. In this investigation, physical mixtures of functionalized and unfunctionalized polymer chains were used to demonstrate the influence of end group on desorption, ionization, and detection probabilities in MALDI-MS. The effect of an end group on a polymer chain also has been investigated by using TOF-SIMS, and comparisons will be made with the same physical mixtures [19].

In this study, two functionalized polymers were investigated: One consists of PS terminated with a dimethylphenylsilyl $\left[-\mathrm{Si}\left(\mathrm{CH}_{3}\right)_{2} \mathrm{C}_{6} \mathrm{H}_{5}\right]$ end group (PS135); the other is PS terminated with a perfluoroalkylsilyl $\left[-\mathrm{Si}\left(\mathrm{CH}_{3}\right)_{2}\left(\mathrm{CH}_{2}\right)_{2} \mathrm{C}_{6} \mathrm{~F}_{13}\right]$ end group (PS405). Each end group functionalized polymer was combined in a 1:1 mole ratio with proton-terminated polymer. In each case, the functionalized polymer and the proton-terminated polymer originated from the same "parent" polymer solution. Therefore, the functionalized and proton-terminated polymers should have the same relative number of chains at each degree of polymerization, but the masses of the chains will be different by the mass of the end group.

As expected, the TOF-MALDI-MS spectra of the mixtures of functionalized and protonated PS are composed of two molecular weight distributions. By ratioing the signals of the functionalized to unfunctional-

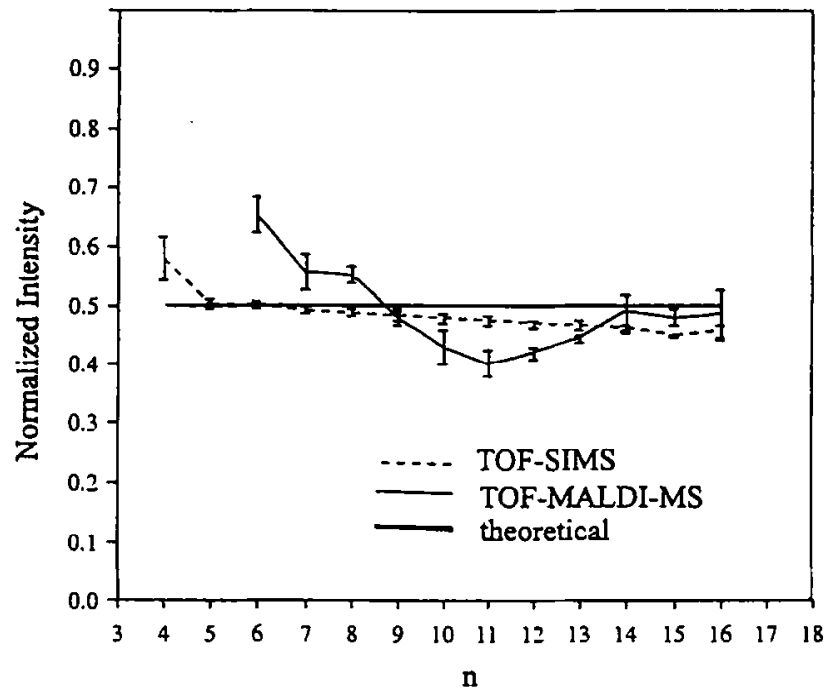

Figure 7. Comparison of relative ion yields of PS-135 oligomers by using TOF-MALDI-MS and TOF-SIMS. (Physical mixture in a 1:1 ratio with $\mathrm{H}$-terminated PS.)

ized oligomers at each degree of polymerization, the effect of the end group on ion yield can be investigated. The values of the ratios versus degree of polymerization are shown in Figures 7 and 8 . A value of 0.50 is expected if the ion yield of functionalized and protonated PS are the same [intensity values are normalized, functionalized PS/(functionalized PS + protonated PS)]. For both types of functionalized PS, the ratio value across all degrees of polymerization is close to 0.50 (values and error bars reflect measurements from three samples). However, the value is not constant across all degrees of polymerization: it is at a minimum in the center with higher values at the high and low mass ends. In accordance with these values, the functionalized oligomers appear to have a broader

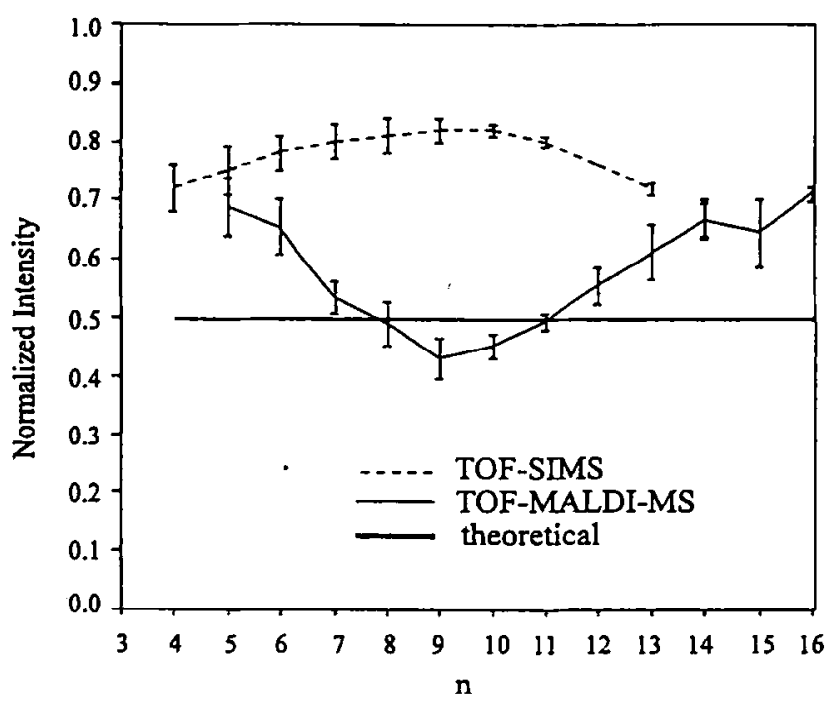

Figure 8. Comparison of relative ion yields of PS- 405 oligomers by using TOF-MALDI-MS and TOF-SIMS. (Physical mixture in a 1:1 ratio with $\mathrm{H}$-terminated $\mathrm{PS}$.) 
polydispersity than the protonated oligomers in the MALDI spectrum. In actuality, the low and high mass oligomers probably have a higher ion yield than the central mass oligomers. The increase in ion yield is most likely due to the presence of the end group, but a result of two distinct phenomena-one for the higher mass oligomers and one for the lower mass oligomers. The higher ion yield of the higher mass oligomers may result from greater interaction of the functionalized PS with the matrix. For the shorter, lower mass oligomers, the end group is a more significant fraction of the molecule and may allow it to ionize more easily than the protonated oligomers. Despite the minor deviations at the low and high mass ends of the distribution, the averaged value over all degrees of polymerization ( 0.50 for dimethylphenylsilyl-terminated PS, 0.58 for perfluoroalkylsilyl-terminated PS) is close to the actual value of the physical mixture of 0.50 .

The results by TOF-SIMS are different from those generated by TOF-MALDI-MS. For the dimethylphenylsilyl functionalized PS, the ratio values are nearly constant over all degrees of polymerization. Furthermore, the average value of 0.48 agrees quite well with the actual value $(0.50)$ and the average value generated by TOF-MALDI-MS (0.50). This suggests that the dimethylphenylsilyl end group does not play a significant role in the ionization of PS oligomers via TOF-SIMS or TOF-MALDI-MS. For the perfluoroalkylsilyl functionalized PS, however, the ratio value (average value is 0.77 ) is higher and varies slightly across all degrecs of polymerization. The high value of the ratio might suggest that the presence of the end group increased the ionization probability of the oligomers. However, given the TOF-MALDI-MS results, where the curvature of the line in the plot has an opposing trend, a different cause is indicated. The surface specificity of the TOF-SIMS technique is thought to play a role in the apparent increase in ion yield. Because the perfluoroalkylsilyl end group has a low surface energy, perhaps the functionalized oligomers may predominate on the surface of the sample (assumes islands of polymer of several monolayers form in a monolayer sample prep). Thus, with TOFSIMS the functionalized oligomers would be more likely to be desorbed, which results in a higher intensity of these ions. With MALDI-MS, the analyte is encompassed by matrix and the technique probes more into the bulk of the sample. In this study, MALDI-MS gives a slightly more accurate quantitative representation of the stoichiometric composition of the physical mixtures, particularly when the average ion yields over all degrees of polymerization are evaluated.

\section{Summary}

A protocol for the preparation of polymeric samples for MALDI-MS analysis has been developed. Optimal quantities of polymer, matrix, and cationization agent were established specifically for PS. Dithranol was identified as the best matrix for PS, and the addition of $\mathrm{Ag}$ for cationization of molecules was necessary. Based on this preparative method, low molecular weight samples of other nonpolar polymers (PI and PBD) were analyzed. Further investigation is necessary to probe higher molecular weight nonpolar polymers, and matrix-polymer interactions. Samples of polar polymers (PEO, PMMA, and PDMS) also were prepared and characterized by using variations of the established protocol.

Polymers up to $49 \mathrm{ku}$ were analyzed. Effects of laser irradiance were found to be most significant for lower molecular weight polymers and skew the distribution of oligomers. Spectra obtained at "threshold" irradiance agreed most closely with the expected distribution of oligomers. The reflectron analyzer was employed for analysis of polymers up to $15 \mathrm{ku}$, with mass resolution for the individual oligomers that was adequate for verification of polymer structure and end group.

TOF-MALDI-MS and TOF-SIMS measurements of the same polymers allowed comparison of the determined molecular weight values and ion yields. The $M_{\mathrm{n}}$ values calculated by TOF-MALDI-MS are consistently higher than values calculated by TOF-SIMS for all classes of polymers that have molecular weights up to $8 \mathrm{ku}$. The molecular weights of the PS calculated from TOF-MALDI-MS are in good agreement with GPC $( \pm 10 \%)$. The role of the terminal group on a polymer chain was determined to have no significant effect on the ion yield of polymer molecules. Initial TOF-SIMS results indicated that the presence of a perfluoroalkylsilyl end group on PS slightly increased its apparent ionization probability. Considering the TOF-MALDI-MS results, the functionalized PS is thought to desorb preferentially from the sample surface due to surface segregation, even for a nominal monolayer preparation, rather than to be ionized preferentially in the TOF-SIMS technique.

\section{Acknowledgements}

We are grateful for the interaction with the J. M. DeSimone polymer group, and especially to Michael Hunt, Jr., Jun Lin, and Mark Peters for synthesis of many of the polymers in this study. We thank Professor A. Benninghoven for access to the TOF-SIMSII instrument and Klaus Meyer for technical assistance. Funding of this work was provided by Monsanto Corporation and the North Carolina Board of Science and Technology. Access to TOF-MALDI-MS instrumentation was facilitated by P. L. Sherman and F. D. Hileman. We thank E. E. Remsen for helpful discussions.

\section{References}

1. Tanaka, K.; Waki, H.; Ido, Y.; Akita, S.; Yoshida, Y.; Yoshida, T. Rapid Commum. Mass Spectrom. 1988, 2, 151.

2. Danis, P. O,; Karr, D. E.; Mayer, F.; Holle, A.; Watson, C. H. Org. Mass Spectrom. 1992, 27, 843.

3. Bahr, U.; Deppe, A.; Karas, M.; Hillenkamp, F.; Giessman, U. Anal. Chem. 1992, 64, 2866. 
4. Danis, P. O.; Kars, D. E. Org. Mass Spectrom. 1993, $28,923$.

5. Xiong, Y.; Owens, K. G.; Danis, P. O.; Karr, D. E. Proceedings of the 42nd ASMS Conference on Mass Spectrometry, 1994; $\mathrm{p} 980$

6. Kahr, M. S.; Wilkins, C. L. Proceedings of the 41st ASMS Conference on Mass Spectrometry, 1993; $\mathrm{p} 783$.

7. Hunt, M. O., Jr.; Belu, A. M.; Linton, R. W.; DeSimone, J. M. Macromolecules 1993, 26, 4854.

8. Niehuis, E.; Heller, T.; Feld, H.; Benninghoven, A. I. Vac. Sci. Technol. A 1987, 5, 1243.

9. Tung, L. H., I. Appl. Polym. Sci. 1979, 24, 953.

10. Juhasz, P. Notes from ASMS Fall Workshop: Laser Desorption in Biological and Polymer Analysis; Boston, MA, 1993.

11. Hillenkamp, F.; Karas, M.; Beavis, R. C.; Chait, B. T. Anal. Chem. 1991, 63, 1193A.
12. Mattern, D. E.; Hercules, D. M. Anal. Chem. 1985, 57, 2041.

13. Holm, R.; Karas, M.; Voigt, H. Anal. Chem. 1987, 59, 371.

14. Kahr, M. S.; Wilkins, C. L. J. Am. Soc. Mass Spectrom 1993, 4, 453.

15. Benninghoven, A.; Hagenhoff, B.; Niehuis, E. Anal. Chem. 1993, 65, 631A.

16. Lacey, M. P.; Keough, T. Proceedings of the 41st ASMS Conference on Mass Spectrontetry, 1993; p 675.

17. Beuhler, R. J.; Friedman, L. Int. J. Mass Spectrom. Ion Phys. $1977,23,81$.

18. Belu, A. M. Doctoral Dissertation, University of North Carolina at Chapel Hill, 1994.

19. Belu, A. M.; Hunt, M. O., Jr.; DeSimone, J. M.; Linton, R. W. Macromolecules 1994, 27, 1905. 